\title{
Insight Into the Crystallinity of Chinese Ancient Silk by Synchrotron Radiation-Based and Conventional X-ray Diffraction Methods
}

\author{
Decai Gong, Xiaoning Zhang, Yuxuan Gong ${ }^{*}$ \\ Basic Research Center of Conservation Science, Department of the History of Science and Scientific Archaeology, University of \\ Science and Technology of China, Hefei 230026, China
}

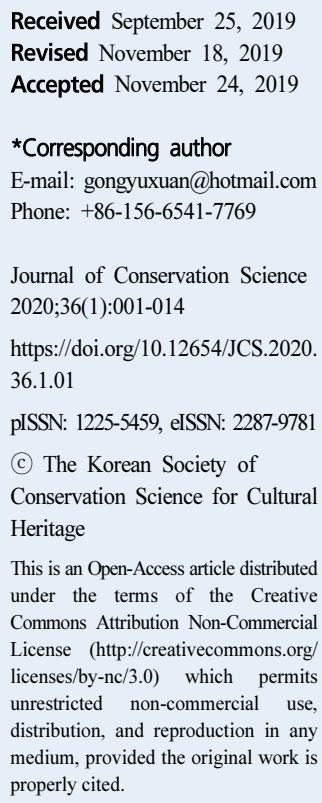

\begin{abstract}
This study investigates the crystallinity distribution of ancient silk. Owing to the inherent multi-hierarchical structure of silk protein and the complicated structural changes that occur due to various burial environments, it is challenging but worthwhile to study ancient silk ageing behavior, which is based on the fact that ageing begins with a single fiber and then spreads to a whole fabric. Crystallinity was one of the most effective indicators found to reveal the ageing status of silk. Therefore, a synchrotron radiation-based X-ray diffraction(SR-XRD) method was employed to study the crystallinity distribution of single fibers of ancient silk unearthed from seven archaeological sites in China from historical periods including the warring states, Han dynasty, Song dynasty, and Ming dynasty. In comparison, the conventional X-ray diffraction method, which uses large amounts of samples, was also performed to determine the integral crystallinity of ancient silk. Thermal stability experiments by thermogravimetry(TG) as well as morphology observations by scanning electron microscopy(SEM) and optical microscopy $(\mathrm{OM})$ all confirmed the deterioration of ancient silk. Moreover, the ageing mechanism of ancient silk was proposed with the assistance of an artificial ageing study. The results confirmed the effectiveness of SR-XRD as an ageing indicator, revealing the crystallinity distribution. This research could provide motivation to determine the deterioration status of ancient silk, and would also aid in explaining the fragility of ancient silk due to ageing.
\end{abstract}

Key Words Ancient silk, Distribution of crystallinity index, Synchrotron radiation-based X-ray diffraction, Conventional X-ray diffraction

\section{INTRODUCTION}

To understand the ageing mechanism of ancient silk is fundamental for silk conservation and preservation. However, owing to the inherent multi-hierarchical structure of silk protein and the complicated structural changes caused by various burial environments, it is challenging but worthwhile to study ancient silk ageing behavior.

Silk is a kind of semi-crystalline material(Koperska et al., 2014). The classic model defines silk as a combination of crystalline and non-crystalline regions. The crystalline region contains closely packed beta-sheet chains with a hydrophobic nature and long-range order. Conversely, the non-crystalline region of silk consists of random coils with a hydrophilic nature and short-range order(Zhou et al., 2001; Ha et al., 2005). Thus, the two phases show distinct chemical properties, which is helpful in our understanding of the ageing of silk.

The crystallinity of silk protein is one of the most important indicators used to trace the ageing of silk. The crystallinity may be investigated through analysis of the beta-sheet secondary structure, indirectly by Fourier transform infrared(FT-IR) spectroscopy(Koperska et al., 2015). Therefore, FT-IR has been widely used as an efficient technique to investigate the microstructural change in aged silk though secondary derivative spectra or the secondary 
structure(Tsuboi et al., 2001; Luo et al., 2012; Liu et al., 2019). X-ray diffraction(XRD) provided a direct method to study the crystallinity of silk. However, conventional XRD methods require large amounts of samples, which must be thoroughly ground into a powder to obtain optimal results. Additionally, ancient silk with a similar integral crystallinity index but with a distinctive distribution could not be distinguished by that method. In recent years, synchrotron radiation-based methods have been increasingly widely used in the study of cultural heritage, from ancient material identification to ageing characterization on very small samples(Martin et al., 2010; Bertrand et al., 2012a; Bertrand et al., 2012b). In comparison with a conventional radiation source, synchrotron radiation offers high-quality spectra on the micro-scale with focused spot size and high beam intensity. The high signal-to-noise XRD patterns are beneficial for further quantitative study and the results have greater reliability(Janssens et al., 2013; Salvadó et al., 2013). Due to the advantages of this technique, sample preparation of ancient silk is not often needed, and the ageing distribution can be revealed from single fibers of silk(Hermes et al., 2006).

In order to better understand the degradation process, evaluate the ageing status, and provide suggestions for the effective preservation of historic or archaeological silk, artificial ageing was often employed to stimulate the ageing process. Artificial ageing was achieved in a controlled environment with ultra violet(UV) radiation, soil embedding, volatile organic compounds(VOC), inorganic gas, animal flesh, or elevated temperature and humidity(Kang, 2009; Baek et al., 2012; Kim et al., 2012; Kim et al., 2013; Kim et al., 2014; Oh et al., 2014; Kim et al., 2016; Park et al., 2018; Park et al., 2019). Among these factors, dry-heat accelerated ageing was adopted in this study, based on the mechanism of ageing starting from a single fiber and then spreading to the whole fabric. In this paper, an synchrotron radiation-based X-ray diffraction(SR-XRD) method was employed to characterize the ageing of ancient silk from seven archaeological sites in south China, combined with thorough morphology studies by scanning electron microscopy(SEM), optical microscopy(OM) and thermal stability experiments by thermogravimetry(TG). A comparison study between the SR-XRD and conventional X-ray diffraction(XRD) of ancient silk was also performed to prove the representativeness of the data from single fibers.
Additionally, in order to clarify the ageing mechanism, artificial ageing of silk was carried out, which aided with tracing the degradation path of ancient silk.

\section{MATERIALS AND METHODS}

\subsection{Materials}

The experiment used Commercial raw B. mori silk fabric(silk, Hefei Guanghua Silk, China), and the chemical reagents(sodium bicarbonate, Sinopharm Chemical Reagent, China). These ancient samples were all unearthed from south China(Figure 1), and were typically submerged in a waterlogged burial environment(Xu et al., 2017). Detailed information on the Chinese ancient silk samples is provided in Table 1.

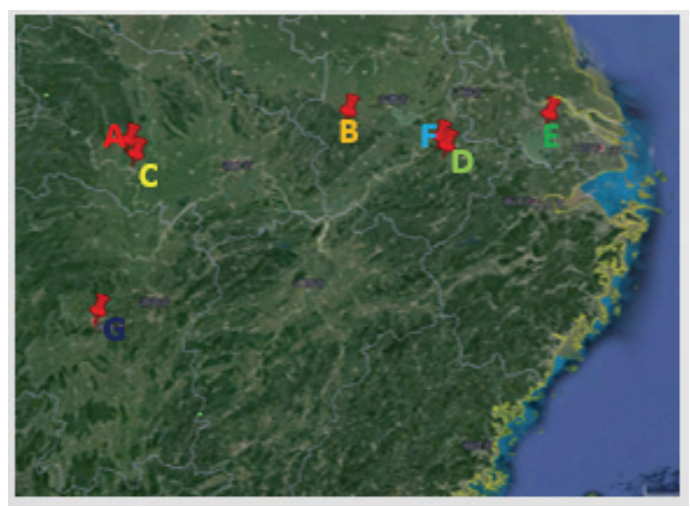

Figure 1. Archaeological sites of the unearthed silk fabrics in China.

\subsection{SR-XRD experiment}

Sample preparation was referred to in our previous study(Zhang et al., 2019). The SR-XRD experiment was carried on Beamline BL15U1 in the Shanghai Synchrotron Radiation Facility in China. Both ends of the thread from fabrics were vertically bonded to the specimen holder. Prior to testing, the sample-to-detector distance was calibrated by $\mathrm{CeO}_{2}$ powder(Fang et al., 2016). Detailed information of the experimental parameters is listed in Table 2. Approximately 30-40 single fibers(except for the Wangshanqiao sample due to the influence of insoluble contaminants) from each ancient silk piece underwent SR-XRD for data representativeness.

Fit2d software was used to record the two-dimensional 
Table 1. Archaeological information of silk samples

\begin{tabular}{|c|c|c|}
\hline Sample number & Sample name & Date \\
\hline A & Wangshanqiao sample & \multirow{2}{*}{ Warring stat } \\
\hline B & Lu'an sample & \\
\hline C & Xiejiaqiao sample & Han dynasty \\
\hline D & Nanling sample & Song dynast. \\
\hline $\mathbf{E}$ & Wuxi sample & \multirow{3}{*}{ Ming dynas } \\
\hline $\mathbf{F}$ & Changzhou sample & \\
\hline G & Hunan sample & \\
\hline $\begin{array}{l}\text { Table 2. Experime } \\
\text { based X-ray diffr }\end{array}$ & $\begin{array}{l}\text { al parameters for synch } \\
\text { ion }\end{array}$ & rotron radiation- \\
\hline \multicolumn{2}{|c|}{ Items } & Parameters \\
\hline \multicolumn{2}{|c|}{ Spot size (KB mirror) } & $5 \times 5 \mu \mathrm{m}^{2}$ \\
\hline \multicolumn{2}{|c|}{ Wavelength } & $0.088 \mathrm{~nm}$ \\
\hline \multicolumn{2}{|c|}{ Exposure time } & $10 \mathrm{~s}$ \\
\hline \multicolumn{2}{|c|}{ Scan range } & $5-30^{\circ}$ \\
\hline \multicolumn{2}{|c|}{ Detector-to-sample distance } & $173.697 \mathrm{~mm}$ \\
\hline
\end{tabular}

diffraction pattern. In order to obtain a quantitative analysis, conversion from the two-dimensional to one-dimensional profiles using azimuthal integration was conducted. The deconvolution process was performed on Grams/AI 9.2, and fresh silk samples were used as control samples for preliminary fitting. The numbers and the position of hidden peaks of fresh silk were referred from the literature(Martel et al., 2007). Due to the possibility that the inorganic salts attached to the ancient silk might contribute to the crystallinity of protein, the crystallinity index was calculated by the following equation rather than Chen's method(Fang et al., 2016), see Eq. 1.

$$
X_{c}=\frac{I_{c}}{I_{c}+I_{a}} * 100 \%
$$

Ic is the sum of the integrated intensity of the (200) and (210) Bragg peaks, and Ia is the integrated intensity of the amorphous halo.

The one-dimensional Gaussian profile was drawn as Q $\left(\mathrm{nm}^{-1}\right)$ against intensity. However, the literature often employs $\mathrm{d}(\mathrm{nm})$ rather than $\mathrm{Q}$. The transformation of $\mathrm{Q}$ to d is expressed by Eq. 4 .

Tomb M1, Wangshan village, Chuandian town, Jingzhou district, Jingzhou city, Hubei province

Jingkai district, Lu'an city, Anhui province

Qinghe village, Guanju town, Shashi district, Jingzhou city, Hubei province

Tieguai Tomb M1, Nanling county

Taodianqiao, Jiangxi commune, Wuxi city, Jiangsu province

Huaide south road, Changzhou city, Jiangsu province

Changde city, Hunan province 
working distance. The samples were sprayed with gold for electric conduction. Image $J$ software(1.52a) was used to analyze the distribution of fiber fineness(width of single fiber) for fresh and ancient silk. OM was performed on a microscope(VHX-2000C, Keyence, USA).

\subsection{Thermal stability}

Powders of fresh and ancient silk samples were analyzed by thermogravimetric analysis(TGA Q5000IR, TA Instruments, USA). The experimental temperature was set to increase from room temperature to $600.00^{\circ} \mathrm{C}$ at a heating rate of $15.00^{\circ} \mathrm{C} / \mathrm{min}$ under nitrogen-gas dynamic atmosphere. The deconvolution of the DTG curve was carried out by GRAMS/AI 9.2, in order to analyze the decomposition rate of aged silk(Hao et al., 2019). The deconvolution method of fresh silk was adopted from the literature(Hao et al., 2019), and the ancient silk was deconvoluted based on the same protocol.
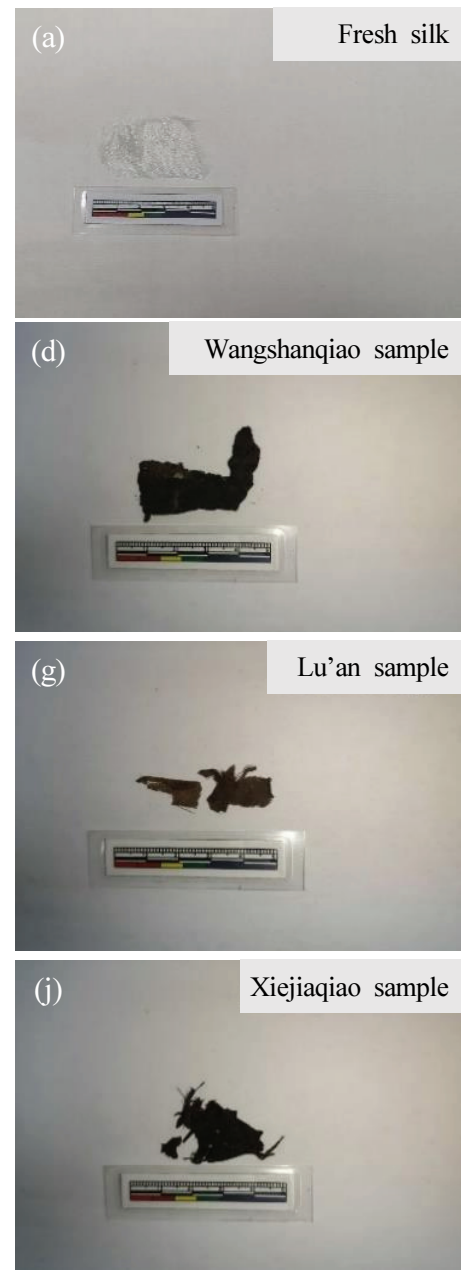
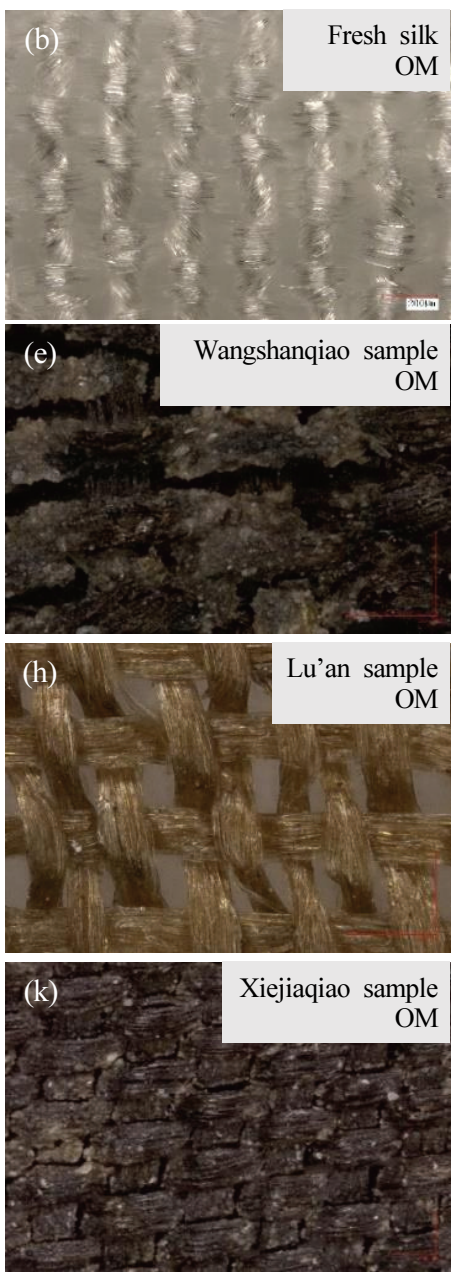
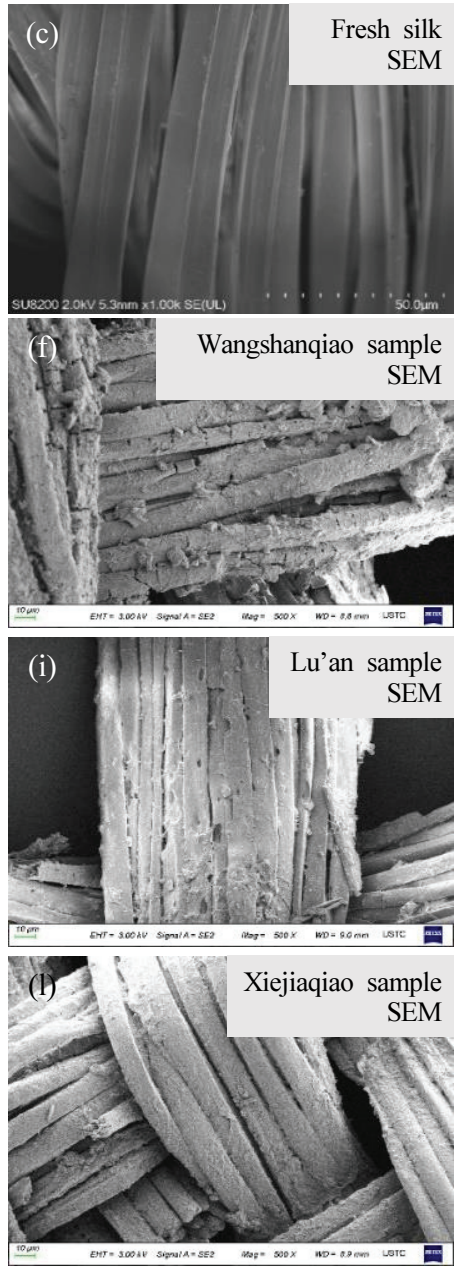
Insight Into the Crystallinity of Chinese Ancient Silk by Synchrotron Radiation-Based ... / Decai Gong, Xiaoning Zhang, Yuxuan Gong | 5
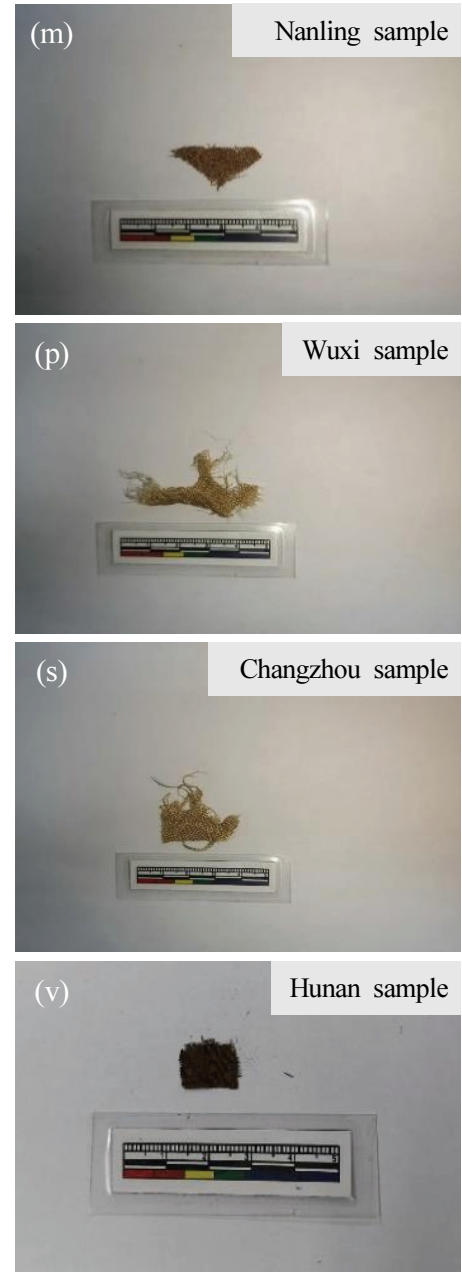
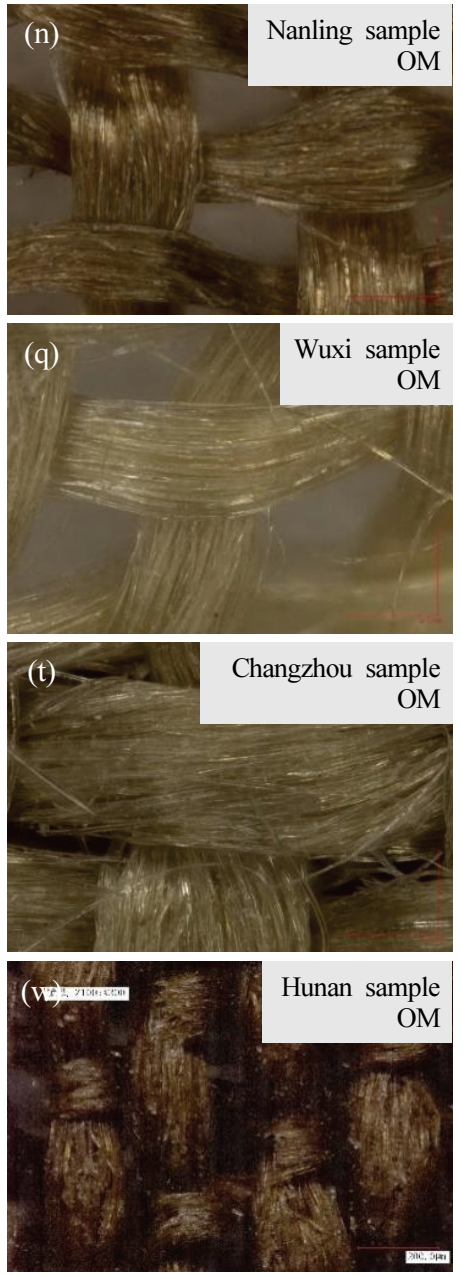
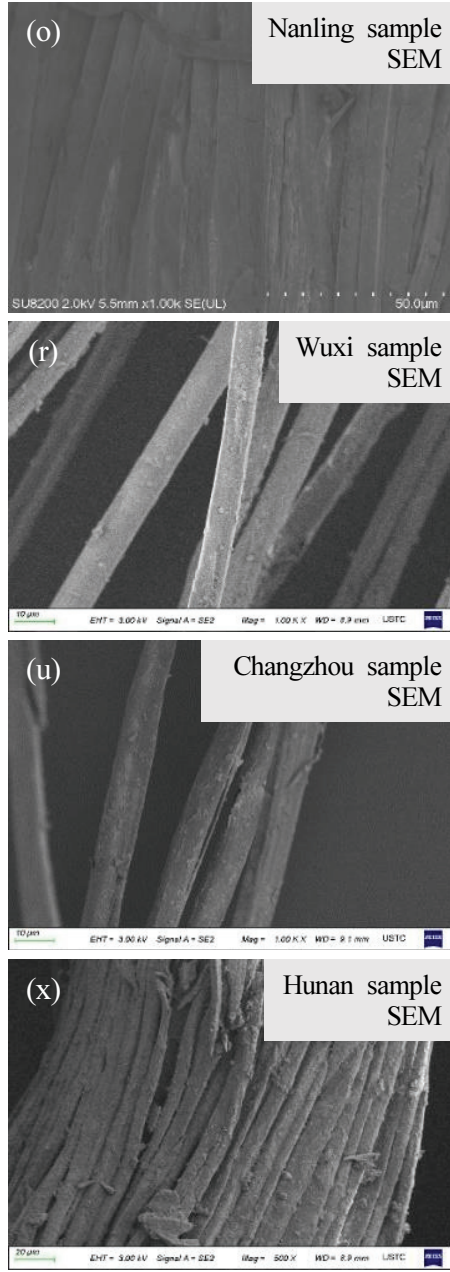

Figure 2. Representative photographs, optical micrographs, and SEM images of ancient silk.

Table 3. Morphology observations of ancient silk

\begin{tabular}{|c|c|c|}
\hline Sample & Morphology observation by OM & Morphology characteristics by SEM \\
\hline Wangshanqiao sample & Stiff and fragile with black color & $\begin{array}{l}\text { Radial cracks or fractures, longitudinal cracks } \\
\text { predominated }\end{array}$ \\
\hline Lu'an sample & Stiff and fragile with brown color & $\begin{array}{l}\text { Large holes on the surface with some } \\
\text { longitudinal cracks and fibrillation }\end{array}$ \\
\hline Xiejiaqiao sample & Stiff and fragile with black color & Longitudinal cracks predominated \\
\hline Nanling sample & Stiff and fragile with brown color & Some cracks and peeling \\
\hline Changzhou sample & Flexible with light yellow color & Smooth surface with few contaminants \\
\hline Wuxi sample & Flexible with light yellow color & Smooth surface with few contaminants \\
\hline Hunan sample & Stiff and fragile with dark brown color & Longitudinal cracks, peeling, and fibrillation \\
\hline
\end{tabular}

The distribution of the fiber fineness of the Wuxi, Changzhou, and Nanling samples was approximately in the range of 6-10 $\mu \mathrm{m}$ (Figure 3), while those of samples unearthed from Lu'an, Xiejiaqiao and Wangshanqiao sites were distributed in the range of 10-14 $\mu \mathrm{m}$. It was possible that the severely degraded samples might be more susceptible to pressure, causing flattening during ageing. This is probably due to the loss of the crystalline region, which was often 


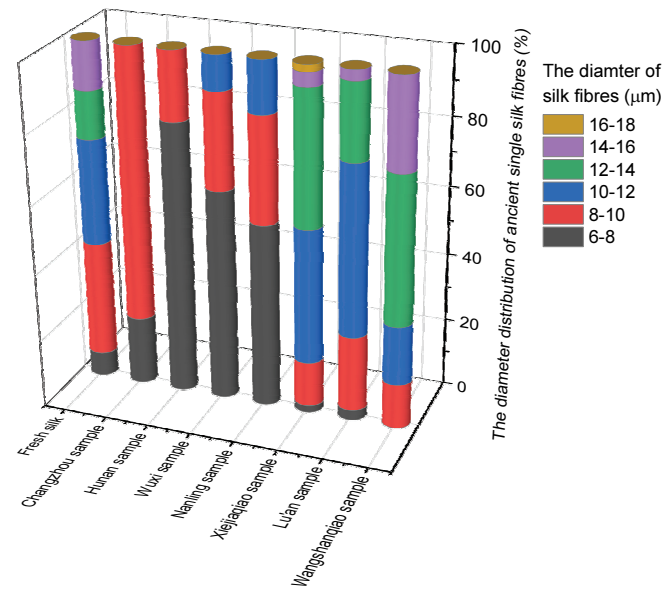

Figure 3. Distribution of fiber fineness of ancient silk.

thought to be associated with the rigidity of silk(Li et al., 2013). Therefore, it confirmed that the distribution of fiber fineness of ancient silks was related to their morphology deterioration, and could serve as a rapid and approximate assessment of silk ageing.

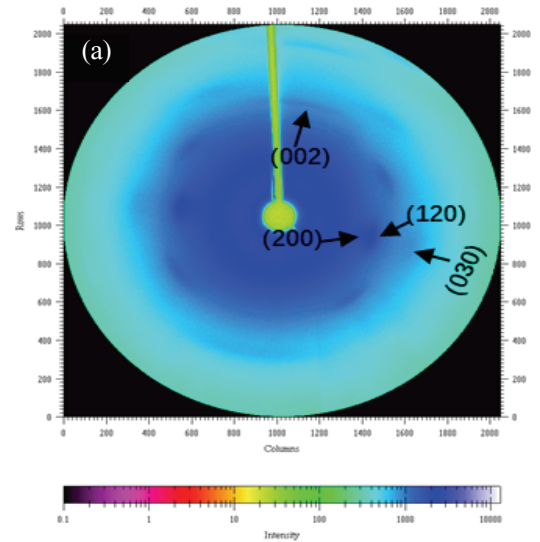

\subsection{Distribution of crystallinity by $S R-X R D$}

\subsubsection{Fresh silk}

The representative 2D-WAXD pattern and the corresponding 1D deconvolution results of fresh silk are shown in Figure 4.

It was demonstrated that the 2D-WAXD pattern was typical of silk protein(Riekel and Vollrath, 2001; Martel et al., 2007; Xu et al., 2015). The diffraction speckle with a very high intensity located near the equator line should belong to crystal planes (200) and (120). The weaker speckle located away from (200) and (120) was (030), and the meridian speckle mainly corresponded to (002). (200) and (120) were not completely laid in the equator line due to the placement of the single fiber; nevertheless, this would not affect the crystallinity study. From the deconvolution results shown in Table 4, both (200) and (120) corresponded to crystalline regions composed of beta-sheet chains with long-range order. The amorphous region indicated by a

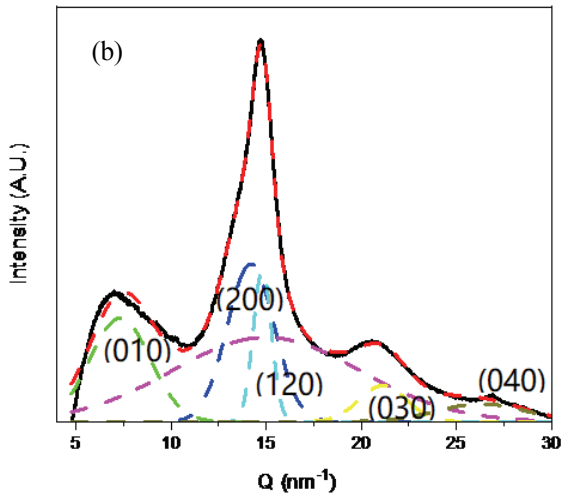

Figure 4. Representative 2D-WAXD of fresh silk. (a) Deconvolution results by using azimuthal integration in Fit2d software, (b) Black line, original spectra; red dashed curve, simulated spectra from summed peaks; other dashed curve, the decomposed components.

Table 4. Deconvolution results of fresh silk by SR-XRD

\begin{tabular}{ccccc}
\hline & $\mathrm{d}(\mathrm{nm})$ & & \\
\cline { 1 - 2 } Our results & Ref & Assignment & Relative area $(\%)$ \\
\hline 0.86 & (Martel et al., 2007; Guo et al., 2018) & $(010)$ beta-sheets & 16.9 \\
0.44 & 0.98 & $(200)$ beta-sheets & 21.3 \\
0.42 & 0.47 & $(120)$ & 8.0 \\
0.42 & 0.43 & Short-range order & 44.5 \\
0.30 & 0.41 & $(030)$ & 5.0 \\
0.24 & & $(040)$ & 4.3 \\
\hline
\end{tabular}


diffusion halo centered at $0.42 \mathrm{~nm}\left(15.0 \mathrm{~nm}^{-1}\right)$ was thought to possess short-range order. The crystallinity of fresh silk was $34.60 \pm 4.00 \%$, which was fairly consistent with XRD study(Sampath et al., 2012).

\subsubsection{Ancient silk}

The representative 2D-WAXD and corresponding 1D-WAXD profiles of ancient silk with deconvolution results are shown in Figure 5. The distributions of the crystallinity index of fresh and ancient silk are displayed in Figure 6.

Figure 6 shows that the proportion of the crystallinity index within $26-42 \%$ predominated for the Wuxi and Changzhou samples. Combined with SEM observations, this indicates that the Wuxi and Changzhou samples were preserved well, compared with other ancient silk samples, by this indicator. For the Hunan sample, a significantly increased ratio of crystallinity at $42-70 \%$ was observed. This phenomenon suggests that the amorphous region of the Hunan sample suffered from more deterioration than the Changzhou and Wuxi samples. Meanwhile, there is a remarkable proportion of crystallinity distributed at $14-26 \%$, suggesting that partial degradation of the crystalline region of the Hunan sample occurred. Compared with the Hunan sample, the Nanling sample showed a lower proportion of crystallinity(42-70\%), indicating less deterioration of the amorphous region. The Hunan sample and Nanling samples
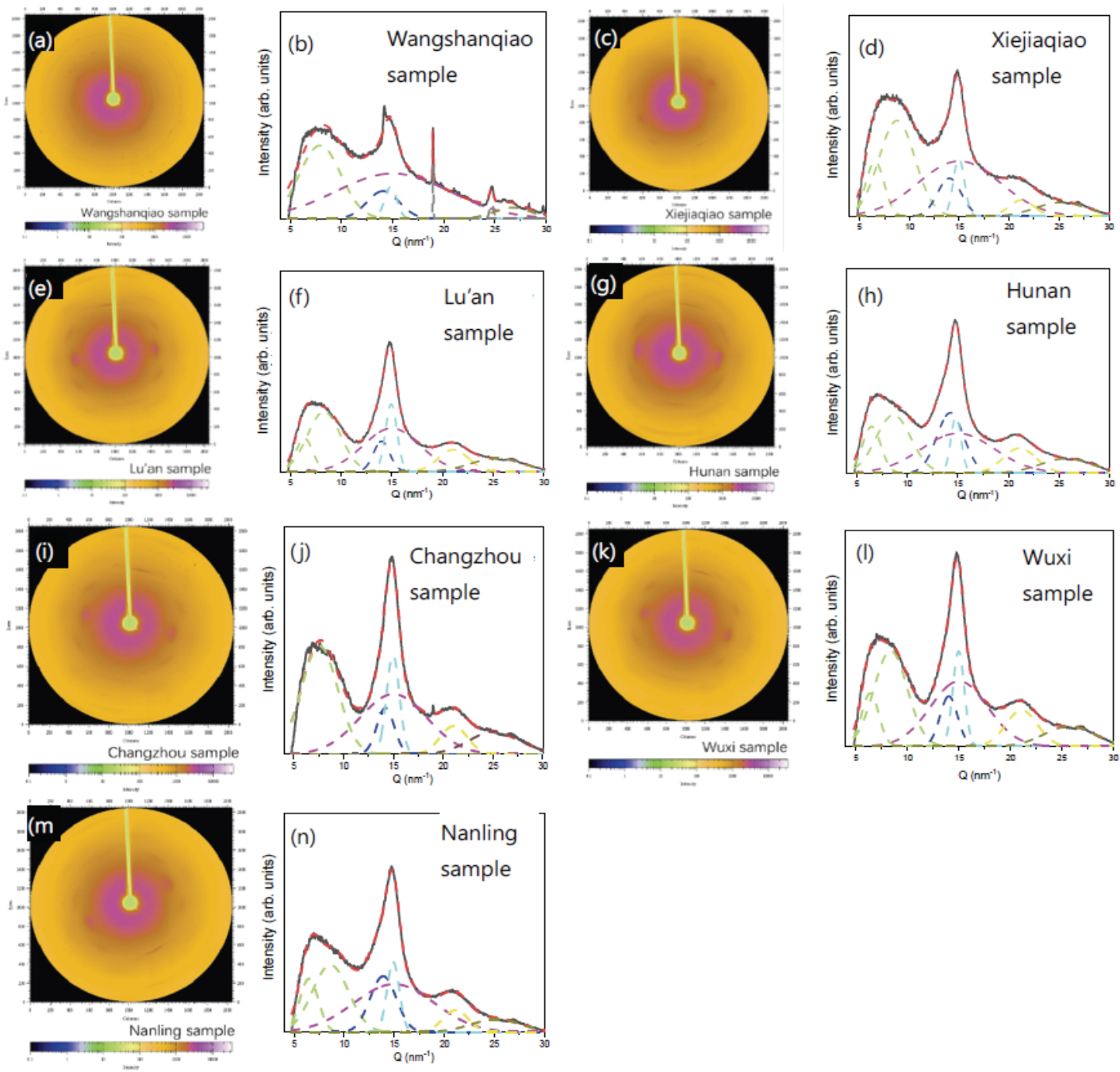

Figure 5. Representative 2D-WAXD and corresponding 1D-WAXD profiles of ancient silk with deconvolution results(the 1D-WAXD pattern was obtained using azimuthal integration in Fit2d software, the black line represents the original spectra, red dashed curves represent the sum of fitting, and other dashed curves represent the decomposed components). 


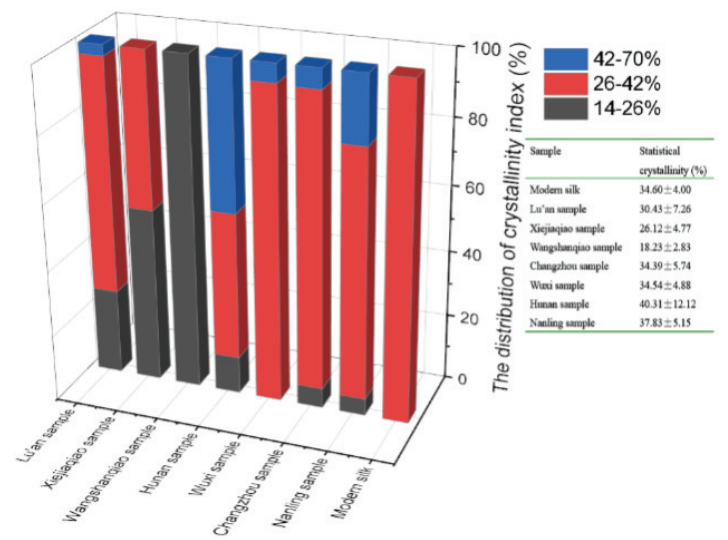

Figure 6. Distribution of crystallinity index of ancient silk with statistical crystallinity inserted.

exhibited similar statistical crystallinity; nevertheless, their ageing condition can be distinguished by their crystallinity distribution.

Notably, the Xiejiaqiao and Lu'an samples showed an increased ratio of crystallinity(14-26\%), indicative of the severe damage of the sample. Likely due to the transformation of crystalline regions into disordered states, the statistical crystallinities of the Xiejiaqiao and Lu'an samples were lower than that of fresh silk. The crystalline region of silk was reported to degrade rapidly in the final stage of ageing by many artificial and natural ageing studies(Greiff et al., 2005; Li et al., 2013; Badillo-Sanchez et al., 2018). Therefore, the crystallinity distributions of the Xiejiaqiao and Lu'an samples suggest that severe degradation occurred, resulting in fragility and a lack of physical strength.

Moreover, the Wangshanqiao sample exhibited approximately $100 \%$ distribution in $14-26 \%$ crystallinity, suggesting almost total degradation of beta-sheet crystallites. The beta-sheet crystallites mainly transformed from long-range order to short-range order. Therefore, the ageing of the Wangshanqiao sample was found to be the greatest among all the ancient samples. In addition, the ancient silk showed increased standard deviation(except for the Wangshanqiao sample) of the crystallinity distribution against fresh silk, indicative of the intrinsic heterogeneity of natural aged silk. Furthermore, compared with the crystallinity distribution of these ancient silks, it seemed that the preservation condition was not only associated with burial time, but also with burial environment.

From the above study, it can be deduced that the distribution of crystallinity was associated with the deterioration conditions of crystalline and amorphous regions in ancient silk, and that the serious deterioration of crystalline regions was associated with the significant loss of physical strength. These assumptions were mostly in agreement with the morphological characteristics determined by SEM.

Microscopic observation is a simple and rapid but significant method to examine the morphology of ageing and the contaminates of fibers in an archaeological excavation site or in the lab. However, this technique is unable to reveal the microstructure, and hence, the factors responsible for the formation of fragile fibers. SR-XRD is a powerful method to investigate the microstructural change in ancient silk with only single fibers; however, SR-XRD experiments require large facilities, and it is impossible to conduct it at an archaeological excavation site.

\subsection{Conventional XRD of artificially aged silk}

In order to trace the degradation path and clarify the ageing mechanism of ancient silk, thermally aged silk fabrics were prepared and underwent conventional XRD. The deconvoluted results of fresh silk and thermally aged silk subject to $1,3,5,7$, and 9 days of heating are presented in our previous study(Zhang et al., 2019). For understanding of the ageing behavior of seriously deteriorated silk, accelerated-age silk with more ageing time was prepared, and subjected to $11,13,15$, and 22 days of dry heat.

From Figure 7, there were four Bragg peaks $\left(\sim 10^{\circ}, \sim 19^{\circ}\right.$, $\sim 21^{\circ}, \sim 25^{\circ}$ ) for beta-sheet crystallites and a broad amorphous halo $\left(\sim 22^{\circ}\right)$ arising from the crystallites with smaller sizes through the XRD pattern of aged silk(Drummy et al., 2007).

Combined with the crystallinity obtained from fresh and thermally aged silk with 1, 3, 5, 7, and 9-day dry-heat ageing (data not shown), the plots of crystallinity against the ageing time are displayed in Figure 7e. The amorphous region of silk initially degraded as polypeptide chains broke apart, resulting in a crystallinity increase when comparing fresh silk to that which was aged 3 days. Probably at that time, degradation of the crystalline region commenced and the increase in the degradation rate led to the decline in crystallinity. At 7 days of heating, the crystalline and amorphous regions reached a similar deterioration speed, which was evidenced by the similarity in crystallinity when compared to fresh silk. From 1 to 7 days ageing, loosening 

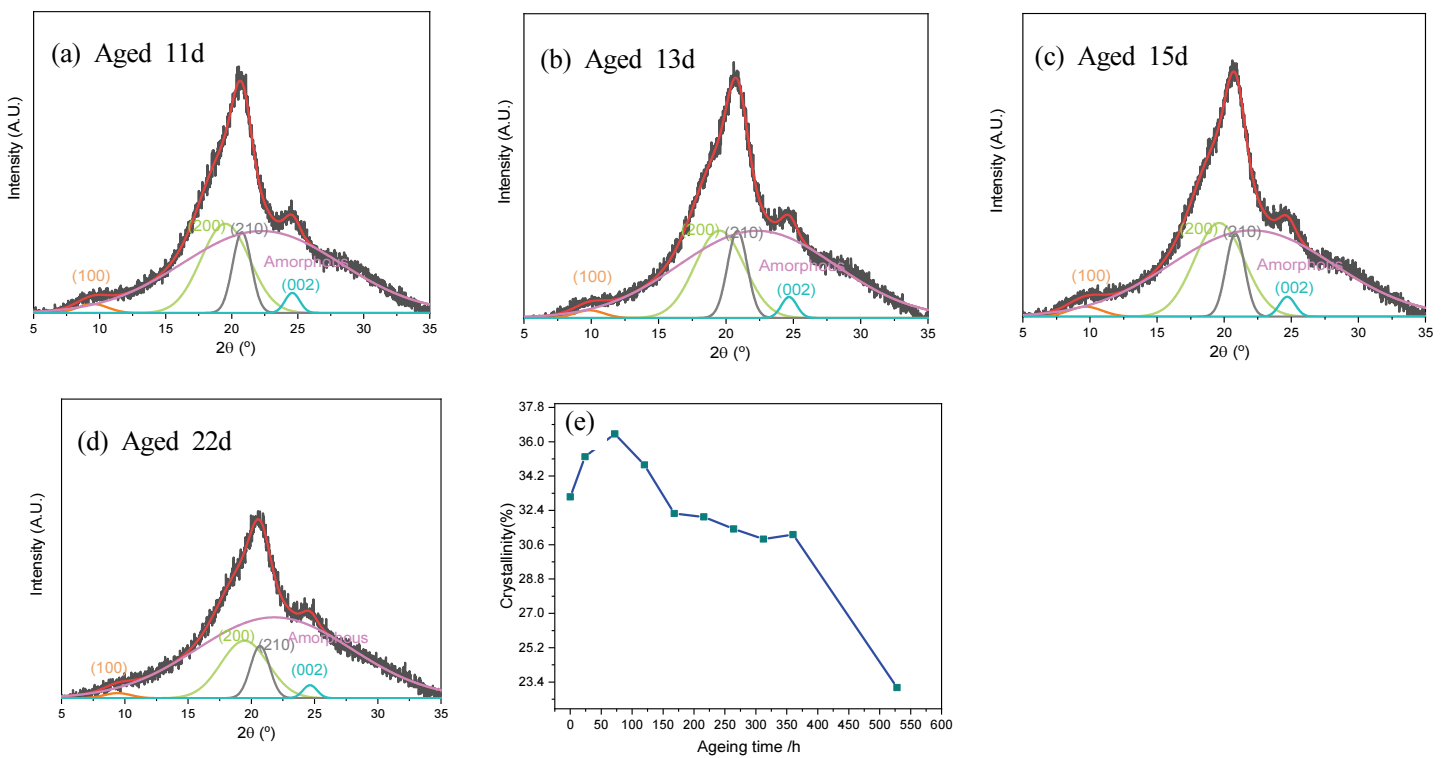

Figure 7. Deconvolution of XRD pattern of aged silk fabrics(a-d, black solid curve, original spectra; red solid curve, simulated spectra from summed peaks; other solid curve, decomposed components), and the plots of crystallinity obtained from deconvolution of XRD patterns(e).

of the closely packed beta-sheet chains occurred, accompanied by a variation in the orientation degree of crystallites as proved by our previous study(Zhang et al., 2019). From 7 to 15 days heating, the crystallinity decreased slowly due to the limited difference in the degradation speed between the crystalline and amorphous regions. From 15 to 22 days ageing, the drastic loss of crystallinity suggested that the crystalline region remarkably transformed into disordered states and scissoring of polypeptide chains may even occur. In this case, the silk was so fragile that it could be easily turned into powder with the highest degradation level.

Although the correlation of the crystallinity with ageing time up to 7 days was not distinct, this could reflect the ageing mechanism of silk. Moreover, the dramatic loss of crystallinity from 15 to 22 days heating suggests that the crystallinity in this range could be employed as a marker to identify seriously degraded silk.

\subsection{Conventional XRD of ancient silk}

To prove the representativeness of the data acquired from single fibers by SR-XRD, results from the conventional XRD using large quantities of ground powders were investigated additionally. Deconvolution was undertaken to obtain the crystallinity of ancient silk, and the results are shown in
Figure 8 .

The integral crystallinity index of the Wuxi and Changzhou samples revealed by conventional XRD was very close to that of fresh silk, and also agreed with the SR-XRD results. The crystallinity of the Nanling sample was higher than that of fresh silk, also in accordance with the SR-XRD study. However, the complete correlation of the crystallinity index with ageing time was not proved by the thermal aging experiment. Combined with the morphology study, it indicated that the Wuxi and Changzhou samples were preserved in good condition compared to the Nanling sample. For the Hunan sample, crystallinity was the highest among all the ancient silks, which was in agreement with the SR-XRD study. Therefore, conventional and SR-XRD methods both confirmed that serious degradation of the amorphous region occurred in the Hunan sample.

For the Xiejiaqiao sample, both XRD studies confirmed that severe deterioration occurred in the crystalline and amorphous regions as evidenced by a remarkably reduced crystallinity index. Due to the extremely low crystallinity of the Wangshanqiao sample, the fitting program could not proceed, implying the highest level of deterioration.

However, the ageing of the Lu'an sample is complicated. SR-XRD demonstrated that the statistical crystallinity was lower than that of fresh silk, while the integral crystallinity 

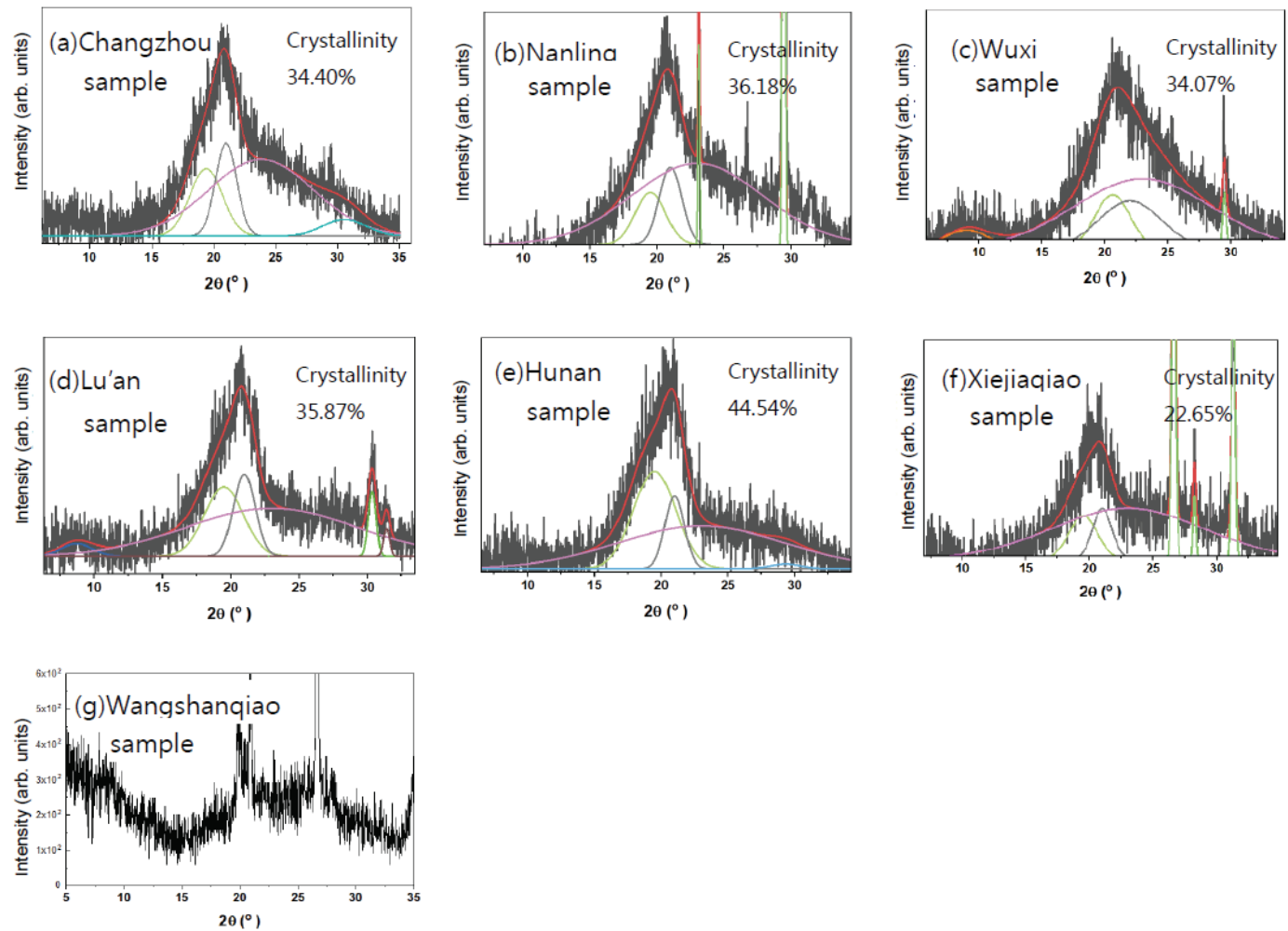

Figure 8. Deconvolution of conventional XRD patterns of ancient silk(black line represents original spectra, red line represents simulated spectra from summed peaks, and other solid curves represent the decomposed components).

was higher when analyzed by conventional XRD. This contradiction could be ascribed to the heterogeneous ageing of the Lu'an sample. When considered in accordance with the fiber fineness distribution study, it was predicted that the deterioration degree of the Lu'an sample would be higher than that of the Hunan sample, but lower than that of the Xiejiaqiao sample. In addition, except for the Lu'an sample, the crystallinity distribution obtained from single fibers by non-destructive SR-XRD and the integral crystallinity from large quantities of powders by conventional XRD were basically consistent. Therefore, the ageing of single fibers could represent the degradation of partial piece of fabric taken from integral piece of ancient silk fabric if the collected single fibers were in sufficiently large quantity.

Furthermore, SR-XRD showed a minor contribution of crystallinity distributed in $14-26 \%$ for the Changzhou and Nanling samples, while conventional XRD was unable to offer these details. It suggested that SR-XRD could trace the serious deterioration process occurring in single fibers of ancient silk, despite the whole fabrics being in fairly good condition.

\subsection{Thermal stability of ancient silk}

TG curves and the deconvolution results of fresh and ancient silk from DTG curves are shown in Figure 9.

The thermal stability experiment showed that the maximum decomposition temperature( $(\mathrm{Tp})$ of ancient silk was far higher than that of fresh silk. The reasons could be ascribed to the integral contribution of the variation in crystallinity, degree of orientation, and the absorbed inorganic salts(Zhang et al., 2019), although the increased proportion of remaining residue from ancient silk was associated with insoluble contaminants, perhaps inorganic materials. Combined with the morphology and crystallinity distribution study, we speculated that the mass loss at $\mathrm{Tp}$ was associated with ancient silk degradation processes to a certain extent.

The deconvolution results of fresh silk showed that there were two main decomposition processes, centered at $\sim 330^{\circ} \mathrm{C}$ and $\sim 400^{\circ} \mathrm{C}$ respectively, which was in accordance with Tong's report(Hao et al., 2019). The full width at half maxima(FWHM) of Tp for ancient silks were all lower than that of fresh silk, suggesting that the decomposition rate of 

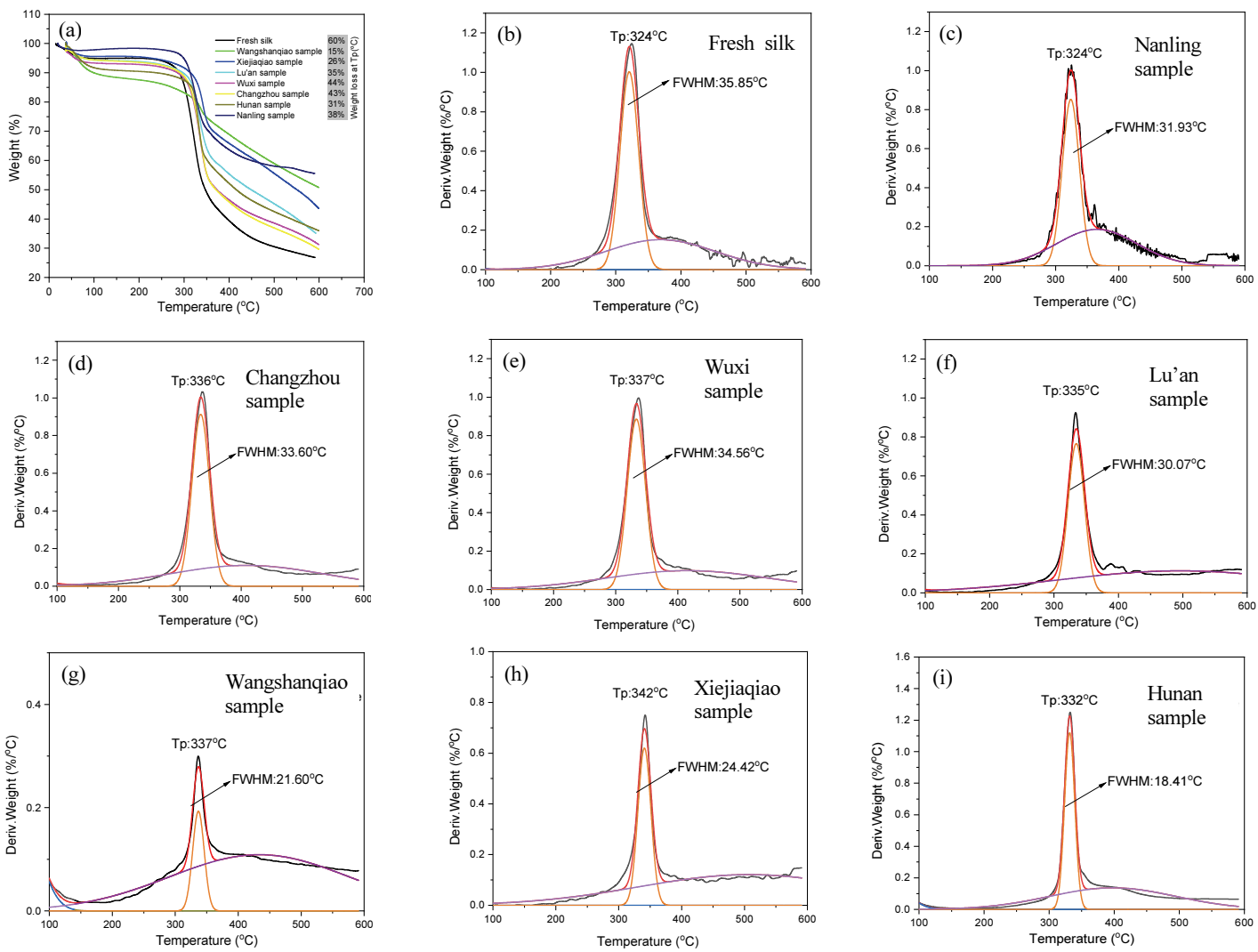

Figure 9. TG curves and the deconvolution results of fresh and ancient silk from DTG curves.

the polypeptide backbone structure of proteins was increased. The Changzhou, Wuxi, and Nanling samples exhibited higher thermal stability than the Xiejiaqiao and Wangshanqiao samples, as evidenced by the remarkably higher FWHM at approximately $330^{\circ} \mathrm{C}$. Therefore, the TG-DTG curve proved that the backbone structures of the Xiejiaqiao and Wangshanqiao samples were deteriorated seriously.

The ageing of the Hunan and Lu'an samples seemed more complicated. The correlation of the degradation condition in amorphous and crystalline regions with the thermal stability revealed by the decomposition rate was not clear. It was probably due to the that the more heterogenous characteristic of these samples than other ancient silks. The degradation condition was complicated even in partial piece of those fabrics with small sample size.

Therefore, results from the thermal stability study of ancient silks were approximately in agreement with SEM observations and crystallinity examinations by SR-XRD and conventional XRD.

\subsection{Ageing mechanism}

Figure 10 shows the ageing mechanism of ancient silk in three different deterioration status. The amorphous region of ancient silk initially swelled by underground water, due to the loose alignment of random coils with hydrophilic amino side chains. Small molecules such as acids and bases attack the random coil, causing scissoring of polypeptide chains and the loss of non-crystalline regions(Li et al., 2013). This phase was proved by the studying the artificially aged silk from fresh to 3 days, and also matched the microstructural characteristic of the ancient sample unearthed from Wuxi, Changzhou, and Nanling sites.

Accompanied with the ageing of the non-crystalline region, the crystalline fraction started to degrade, as revealed by the thermally aged silk at 3 days and the Hunan sample with the highest integral crystallinity. In this phase, the beta-sheet chains within crystalline regions began to undergo conformational and orientational changes, mainly from long-range order to short-range order. Subsequently, the 


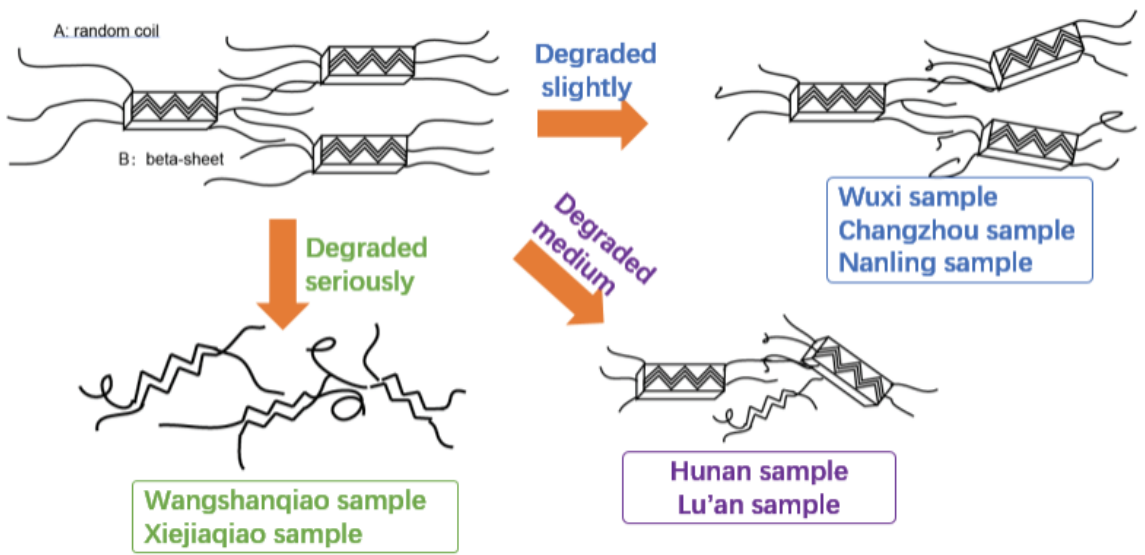

Figure 10. Ageing mechanism of ancient silk with slight, medium, and serious degradation.

degradation of crystalline regions accelerated with the breakage of well-aligned beta-sheet chains from 7 to 15 days heating. This ageing phase behavior was shown by the Lu'an sample. In the late stage of degradation, the crystallinity was almost completely lost and converted to a disordered status with scissoring of polypeptides. This phase was demonstrated by the artificially aged silk during 15 to 22 days heating, and also evidenced by the most deteriorated silks were from Xiejiaqiao and Wangshanqiao.

\section{CONCLUSIONS}

The ancient silk used in this study was unearthed from seven archaeological sites in south China dated from Warring States and the Han, Song, and Ming dynasties. The distribution of crystallinity identified by SR-XRD could explain the degradation status of the amorphous and crystalline regions of ancient silk. Combined with the morphology observations by SEM and OM as well as the thermal stability by TG, it was confirmed that the Wuxi and Changzhou samples from the Ming dynasty were preserved well compared to other ancient silks. After examination by SR-XRD, the Wuxi and Changzhou samples showed a 5-6\% distribution in crystallinity(42-70\%), which was not found in fresh silk. The Nanling sample was more fragile in physical strength compared with the Wuxi and Changzhou samples, and it showed approximately a 20\% distribution in crystallinity(42-70\%). Despite the fact that the number of excavated silk fabrics was limited, based on the study of these ancient samples, the increased distribution amount in
$42-70 \%$ crystallinity could be indicative of the early ageing of ancient silk. For severely damaged silk as evidenced by a physical strength and morphology study, the Xiejiaqiao and Wangshanqiao samples showed a $50-100 \%$ distribution in crystallinity(14-26\%). This confirmed that the initial ageing began in the amorphous region with a remarkably increased distribution in $42-70 \%$ crystallinity. Subsequently, the crystalline region was damaged and transformed into a disordered status, which was reflected by an increased ratio in crystallinity index at $14-26 \%$.

Moreover, the ageing mechanism of ancient silk was elucidated with the help of an artificial ageing study by conventional XRD. Meanwhile, the crystallinity distribution from single fibers of ancient silk pieces by non-destructive SR-XRD was mostly in agreement with that obtained by conventional XRD using large quantities of ground powder. Therefore, SR-XRD was an effective methodology, not only demonstrate that the crystallinity distribution could be used as an indicator for the deterioration assessment, but also to explain the fragility of ancient silk during ageing.

We hope this research could provide warning of the early ageing of ancient silk(excavated silk or historic silk) during conservation and preservation procedures. Second, we anticipate the crystallinity indicator to be used to reveal the microstructural change in severely deteriorated ancient silk, such as powdering or fracturing. In addition, this marker could be potentially applied in reinforcement assessments of ancient silk, especially with biochemical restoration treatment. 


\section{ACKNOWLEDGMENTS}

The SR-XRD data were collected from Beamline BL15U1 at the National Centre for Protein Science in the Shanghai Synchrotron Radiation Facility, China, with the assistance of Mr Jiang Sheng. The SEM images were obtained from Instruments' Center for Physical Science, University of Science and Technology of China, with the help of Mr Zhou Hongmin.

\section{REFERENCES}

Badillo-Sanchez, D., Chelazzi, D., Giorgi, R., Cincinelli, A. and Baglioni, P., 2018, Characterization of the secondary structure of degummed Bombyx mori silk in modern and historical samples. Polymer Degradation and Stability, 157, 53-62.

Baek, Y.M., Goto-Doshida, S. and Saito, M., 2012, Effects of the wet cleaning to the color change of the dyed fabrics with natural dyes. Journal of Conservation Science, 28(1), 21-27. (in Korean with English abstract)

Bertrand, L., Cotte, M., Stampanoni, M., Thoury, M., Marone, F. and Schöder, S., 2012a, Development and trends in synchrotron studies of ancient and historical materials. Physics Reports, 519(2), 51-96.

Bertrand, L., Robinet, L., Thoury, M., Janssens, K., Cohen, S.X. and Schöder, S., 2012b, Cultural heritage and archaeology materials studied by synchrotron spectroscopy and imaging. Applied Physics A, 106(2), 377-396.

Drummy, L.F., Farmer, B.L. and Naik, R.R., 2007, Correlation of the $\beta$-sheet crystal size in silk fibers with the protein amino acid sequence. Soft Matter, 3(7), 877-882.

Fang, G.Q., Huang, Y.F., Tang, Y.Z., Qi, Z.M., Yao, J.R., Shao, Z.Z., and Chen, X., 2016, Insights into silk formation process: Correlation of mechanical properties and structural evolution during artificial spinning of silk fibers. Acs Biomaterials Science \& Engineering, 2(11), 1992-2000.

Greiff, S., Kutzke, H., Riekel, C. and Wyeth, P., 2005, Surveying silk fibre degradation by crystallinity determination: A study on the Tang-Dynasty silk treasure from Famen Temple, China. Scientific Analysis of Ancient and Historic Textiles: Informing Preservation, Display and Interpretation. AHRB Research Centre for Textile Conservation and Textile Studies, First Annual Conference 2004.

Guo, C.C., Zhang, J., Jordan, J.S., Wang, X.G., Henning, R.W. and Yarger, J.L., 2018, Structural comparison of various silkworm silks: An insight into the structureproperty relationship. Biomacromolecules, 19(3), 906-917.

Ha S.W., Gracz, H.S., Tonelli, A.E. and Hudson, S.M., 2005, Structural study of irregular amino acid sequences in the heavy chain of bombyx mori silk fibroin. Biomacromolecules, 6(5), 2563-2569.

Hao, X.Y., Wang, X., Yang, W.M., Ran, J.B., Ni, F.F., Tong, T., Dai, W., Zheng, L.Y., Shen, X.Y., and Tong, H., 2019, Comparisons of the restoring and reinforcement effects of carboxymethyl chitosan-silk fibroin (Bombyx Mori/ Antheraea Yamamai/Tussah) on aged historic silk. International Journal of Biological Macromolecules, 124, 71-79.

Hermes, A.C., Davies, R. J., Greiff, S., Kutzke, H., Lahlil, S., Wyeth, P. and Riekel, C., 2006, Characterizing the decay of ancient Chinese silk fabrics by microbeam synchrotron radiation diffraction. Biomacromolecules, 7(3), 777-783.

Janssens, K., Alfeld, M., Van, d. S.G., De, N.W., Vanmeert, F., Radepont, M., Monico, L., Dik, J., Cotte, M. and Falkenberg, G., 2013, The use of synchrotron radiation for the characterization of artists' pigments and paintings. Annual Review of Analytical Chemistry, 6(1), 399-425.

Kang, D.I., 2009, The stability appraisement on cultural property material with the replacing fumigation gas of methyl bromide. Journal of Conservation Science, 25(3), 283-291. (in Korean with English abstract)

Kim, M.N., Lim, B.A. and Lee, S.M., 2014, Damage characteristics of korean traditional textiles by formaldehyde. Journal of Conservation Science, 30(4), 353-364. (in Korean with English abstract)

Kim, M.N., Lim, B.A. and Lee, S.M., 2016, Damage characteristics of korean traditional textiles by acetaldehyde. Journal of Conservation Science, 32(3), 321-331. (in Korean with English abstract)

Kim, M.N., Lim, B.A., Kim, S. and Lee, S.M., 2013, Damage characteristics of Korean traditional textiles by nitrogen dioxide (NO2) concentrations. Journal of Conservation Science, 29(3), 197-207. (in Korean with English abstract)

Kim, M.N., Lim, B.A., Shin, E.J. and Lee, S.M., 2012, Damage characteristics of korean traditional textiles by sulfur dioxide. Journal of Conservation Science, 28(4), 321-328. (in Korean with English abstract)

Koperska, M.A., Łojewski, T. and Łojewska, J., 2015, Evaluating degradation of silk's fibroin by attenuated total reflectance infrared spectroscopy: Case study of ancient banners from Polish collections. Spectrochimica Acta Part A Molecular \& Biomolecular Spectroscopy, 135, 576-582.

Koperska, M.A., Pawcenis, D, Bagniuk, J., Zaitz, M.M., Missori, M., Łojewski, T. and Łojewska, J., 2014, Degradation markers of fibroin in silk through infrared 
spectroscopy. Polymer Degradation and Stability, 105, 185-196.

Li, M.Y., Zhao, Y., Tong, T., Hou, X.H., Fang, B.S., Wu, S.Q., Shen, X.Y. and Tong, H., 2013, Study of the degradation mechanism of Chinese historic silk (Bombyx mori) for the purpose of conservation. Polymer Degradation \& Stability, 98(3), 727-735.

Liu, H.L., Zhao, S.H., Zhang, Q., Yeerken, T. and Yu, W.D., 2019, Secondary structure transformation and mechanical properties of silk fibers by ultraviolet irradiation and water. Textile Research Journal, 89(14), 2802-2812.

Lu, Y.H., Lin, H., Chen, Y.Y., Wang, C. and Hua, Y.R., 2007, Structure and performance of Bombyx mori silk modified with nano-TiO2 and chitosan. Fibers and Polymers, 8(1), 1-6.

Luo, X.Y., Wu, J.Q., Intisar, A., Geng, J.P., Wu, L.M., Zheng, K.Y. and Du, Y.P., 2012, Study on light aging of silk fabric by Fourier transform infrared spectroscopy and principal component analysis. Analytical Letters, 45(10), 1286-1296.

Martel, A., Burghammer, M., J Davies, R. and Riekel, C., 2007, Thermal behavior of Bombyx mori silk: Evolution of crystalline parameters, molecular structure, and mechanical properties. Biomacromolecules, 8(11), 35483556.

Martin, M.C., Schade, U., Lerch, P. and Dumas, P., 2010, Recent applications and current trends in analytical chemistry using synchrotron-based Fourier-transform infrared microspectroscopy. TrAC Trends in Analytical Chemistry, 29(6), 453-463.

Oh, J.S., Choi, J.E., Noh, S.J. and Eum, S.W., 2014, The effects of anoxic treatments on color and mechanical property in fabrics, natural dyed fabrics, papers, natural dyed papers and paints. Journal of Conservation Science, 30(2), 219-234. (in Korean with English abstract)

Park, H.J., Hwang, M. and Chung, Y.J., 2018, An assessment of the deterioration of fabricated metal thread with light, and temperature and humidity factors: A focused study of Asian textile collections at the Metropolitan Museum of Art, New York. Journal of Conservation Science, 34(4), 245-257.
Park, H.J., Hwang, M. and Chung, Y.J., 2019, Cleaning fabricated metal thread: A post-treatment stability assessment after artificial deterioration and the application of synthetic soil. Journal of Conservation Science, 35(1), 19-31.

Riekel, C. and Vollrath, F., 2001, Spider silk fibre extrusion: Combined wide- and small-angle X-ray microdiffraction experiments. International Journal of Biological Macromolecules, 29(3), 203-210.

Salvadó, N., Butí, S., Cotte, M., Cinque, G. and Pradell, T., 2013, Shades of green in 15th century paintings: Combined microanalysis of the materials using synchrotron radiation XRD, FTIR and XRF. Applied Physics A: Materials Science and Processing, 111(1), 47-57.

Sampath, S., Isdebski, T., Jenkins, J.E., Ayon, J.V., Henning, R.W., Orgel, J.P., Antipoa, O. and Yarger, J.L., 2012, X-ray diffraction study of nanocrystalline and amorphous structure within major and minor ampullate dragline spider silks. Soft Matter, 8(25), 6713-6722.

Tsuboi, Y., Ikejiri, T., Shiga, S., Yamada, K., and Itaya, A., 2001, Light can transform the secondary structure of silk protein. Applied Physics A, 73(5), 637-640.

Xu, D., Shi, X.Y., Thompson, F., Weber, W.S., Mou, Q.S., Yarger, J.L., 2015, Protein secondary structure of Green Lynx spider dragline silk investigated by solid-state NMR and X-ray diffraction. International Journal of Biological Macromolecules, 81, 171-179.

Xu, J.J., Wei, Y.F., Jia, H.Q., Xiao, L. and Gong, D.C., 2017, A new perspective on studying burial environment before archaeological excavation: Analyzing bacterial community distribution by high-throughput sequencing. Scientific Reports, 7, 41691.

Zhang, X.N., Gong, D.C., and Gong, Y.X., 2019, Insight into the orientation behavior of thermal-aged and historic silk fabrics by polarized FTIR microspectroscopy. Journal of Cultural Heritage, 38, 53-63.

Zhou, C.Z., Confalonieri, F., Jacquet, M., Perasso, R., Li, Z.G., and Janin, J., 2001, Silk fibroin: Structural implications of a remarkable amino acid sequence. Proteins-Structure Function and Genetics, 44(2), 119-122. 